\title{
Moving Mass Actuated Reentry Vehicle Control Based on Trajectory Linearization
}

\author{
Xiao-Long Su*, Jian-Qiao Yu**, Ya-Fei Wang*** and Lin-lin Wang**** \\ Key Laboratory of Dynamics and Control of Flight Vehicle, Ministry of Education, School of Aerospace Engineering, Beijing \\ Institute of Technology, Beijing 100081, China
}

\begin{abstract}
The flight control of re-entry vehicles poses a challenge to conventional gain-scheduled flight controllers due to the widely spread aerodynamic coefficients. In addition, a wide range of uncertainties in disturbances must be accommodated by the control system. This paper presents the design of a roll channel controller for a non-axisymmetric reentry vehicle model using the trajectory linearization control (TLC) method. The dynamic equations of a moving mass system and roll control model are established using the Lagrange method. Nonlinear tracking and decoupling control by trajectory linearization can be viewed as the ideal gain-scheduling controller designed at every point along the flight trajectory. It provides robust stability and performance at all stages of the flight without adjusting controller gains. It is this "plug-and-play" feature that is highly preferred for developing, testing and routine operating of the re-entry vehicles. Although the controller is designed only for nominal aerodynamic coefficients, excellent performance is verified by simulation for wind disturbances and variations from $-30 \%$ to $+30 \%$ of the aerodynamic coefficients.
\end{abstract}

Key words: trajectory linearization control (TLC), aerodynamic coefficients variation, roll control system

\section{Nomenclature}

$A(t), B(t), A_{z}(t)$
$x(t)$
$u(t)$
$y(t)$
$\bar{x}(t)$
$\bar{y}(t)$
$\bar{u}(t)$
$e=x-\bar{x}$
$u_{l c}=u-\bar{u}$
$M$

$\mathrm{m}$

$\checkmark$

$P_{b}$

$\begin{aligned}= & \text { state-space system matrices } \\ = & \text { state vector } \\ = & \text { input vector } \\ = & \text { output vector } \\ = & \text { nominal state } \\ = & \text { nominal output trajectories } \\ = & \text { nominal control } \\ = & \text { state error } \\ = & \text { tracking error control input } \\ = & \text { mass of maneuvering re-entry } \\ & \text { vehicle (MaRV) exclusive of } \\ & \text { moving mass } \\ = & \text { mass of moving-mass element } \\ = & \text { velocity of MaRV } \\ = & \text { relative position of mass with } \\ & \text { respect to body coordinate system }\end{aligned}$

F

G

$\rho$

$S$

$\alpha$

$\beta$

$C_{x 0}, C_{x}^{\alpha^{2}}, C_{x}^{\beta^{2}}$

$C_{y 0}, C_{y}^{\alpha}$

$C_{z 0}, C_{z}^{\beta}$

$\omega$

$\vartheta$

$\psi$

$\gamma$

$C_{b}^{g}$
$=$ net aerodynamic force on two- body system
= gravitational force on two-body system
$=$ air density
$=$ characteristic area
$=$ angle of attack
$=$ sideslip angle
$=$ resistance coefficients
$=$ lift coefficients
$=$ lateral force coefficients
$=$ angular velocity
$=$ pitch angle
= yaw angle
$=$ roll angle
$=$ the direction-cosine matrix from body coordinate system to ground coordinate system

This is an Open Access article distributed under the terms of the Creative Commons Attribution Non-Commercial License (http://creativecommons.org/licenses/by$\mathrm{nc} / 3.0 /$ which permits unrestricted non-commercial use, distribution, and reproduction in any medium, provided the original work is properly cited.
(c) * Master Student
** Professor, Corresponding author: jianqiao@bit.edu.cn *** Ph. D Student $* * * * \mathrm{Ph}$. D Student




$\begin{array}{lrl}J & =\text { moment of inertia of MaRV with } \\ & \text { respect to body coordinate system } \\ = & \text { moment of inertia of moving } \\ & \text { mass with respect to body } \\ J_{m} & \text { coordinate system } \\ = & \text { net aerodynamic moment about } \\ & \text { MaRV's center of mass } \\ = & \text { characteristic length } \\ M^{a} & =\text { roll moment coefficients } \\ L & =\text { yaw moment coefficients } \\ m_{x 0}, m_{x}^{\beta}, m_{x}^{\bar{\omega}_{x}}, m_{x}^{\bar{\omega}_{y}}= & \text { pitch moment coefficients } \\ m_{y}^{\beta}, m_{y}^{\bar{\omega}_{y}}, m_{z}^{\bar{\beta}} & =\text { reduced mass parameter } \\ m_{z 0}, m_{z}^{\alpha}, m_{z}^{\bar{\omega}_{z}}, m_{z}^{\bar{\alpha}}= & \text { PD spectrum } \\ \mu & =\text { constant damping } \\ \rho_{i, k}(t)(k=1,2) & =\text { time-varying bandwidth } \\ \zeta_{i}, \zeta & =\text { time-varying parameters } \\ \omega_{n i}(t), \omega(t) & \end{array}$

\section{Superscripts:}

$\begin{array}{ll}\mathrm{g} & =\text { ground coordinate system } \\ \mathrm{b} & =\text { body coordinate system }\end{array}$

\section{Introduction}

Increasing emphasis has been placed on the need for maneuvering re-entry vehicle (MaRV) designs, since future missions for atmospheric re-entry vehicles are facing the problem of a complex environment, short action time, severe weight and volume constraints on actuation and instrumentation. The simplicity of a moving-mass roll control system (MMRCS), combined with its unique ability to provide roll control from within the MaRV's protective shell, make it an attractive alternative to more traditional aerodynamic or thruster-based roll control systems [1]. The purpose of this paper is to present the roll controller using trajectory linearization control (TLC) method that can handle the uncertainties in disturbances and modeling of many modern control problems as exemplified by the controller for an MaRV.

The governing equations of motion of a coupled MaRVmoving mass two-body system are derived using the Lagrange method $[2,4,5]$. The mathematical model has a clear physical meaning and is free from force analysis. Classical control theories, such as PID, can barely meet the needs of MMRCS due to the nonlinearity, coupling and time-varying characteristics of the mathematical model. So modern control methods, such as optimum control [1], quadratic programming [5], and $H_{\infty}$ control [6] are widely used in designing MMRCS. Although the results of the modern control methods are very attractive, the methods are unsuitable for engineering application. For example, an accurate mathematical model is essential for most modern control methods. However, the uncertainties in disturbances and modeling of an actual system may lead to degradation or failure in the controller.

TLC is an effective nonlinear control method and it has been successfully applied in the control systems of missiles $[7,8,10]$, robots [12] and X33 vehicle[13]. The design procedure of TLC consists of the design of two controller subsections. The first one is designed to put the vehicle on the desired trajectory by inverting the nonlinear plant. The second one is a PD-spectrum assignment controller that exponentially stabilizes the linearized tracking error dynamics. This method provides closed-loop global exponential stability without disturbances and gains the maximum robustness against disturbances. The original mathematical model of the nonlinear system is not suitable for analysis, because TLC is based on affine nonlinear systems. So the simplified roll channel dynamic equation derived from the original mathematical model is used to design the controller. Although the controller is designed only for nominal aerodynamic coefficients, excellent performance is verified by simulation for wind disturbances and variations from $-30 \%$ to $+30 \%$ of the aerodynamic coefficients.

\section{Trajectory Linearization}

Suppose the nonlinear system is described by

$$
\begin{aligned}
& \dot{x}(t)=f(x(t))+g(x(t)) u(t) \\
& y(t)=h(x(t))
\end{aligned}
$$

where $x(t) \in \mathbb{R}^{n}, u(t) \in \mathbb{R}^{p}, y(t) \in \mathbb{R}^{m} . f(x(t)), g(x(t))$ are sufficiently smooth known vector fields of time that are bounded, and have bounded, continuous derivatives up to (n - 1) times. $h(x(t))$ is a smooth known function. Let $\bar{x}(t), \bar{y}(t), \bar{u}(t)$ be the nominal state, nominal output trajectories and nominal control satisfying

$$
\begin{aligned}
\dot{\bar{x}}(t) & =f(\bar{x}(t))+g(\bar{x}(t)) \bar{u}(t) \\
\bar{y}(t) & =h(\bar{x}(t))
\end{aligned}
$$

Define the state errors and the tracking error control input by $e=x-\bar{x}, u_{l c}=u-\bar{u}$. Then the tracking error dynamics are governed by

$$
\begin{aligned}
\dot{e} & =f(\bar{x}+e)+g(\bar{x}+e)\left(\bar{u}+u_{l c}\right)-f(\bar{x})-g(\bar{x}) \bar{u} \\
& =F\left(\bar{x}, \bar{u}, e, u_{l c}\right)
\end{aligned}
$$

Asymptotic tracking can then be achieved by a 2 Degree- 
of-Freedom (DOF) controller consisting of: (i) a dynamic inverse I/O mapping of the plant to compute the nominal control function $\bar{u}$ for any given nominal output trajectory $\bar{y}(t)$, of which there is a detailed discussion in $\operatorname{Ref}[11]$ about the pseudo-inverse and the non-minimum phase case and (ii) a tracking error stabilizing control law $u_{l c}$ to account for modeling simplifications and uncertainties, disturbances and excitation of internal dynamics $[9,14]$. For the unperturbed system of Equation (3), exponential stability is the strongest robustness with respect to all kinds of perturbations, and it guarantees finite gain boundedinput-bounded-output stability. The structure of TLC control is illustrated in Fig. 1.

Since nominal state $\bar{x}(t)$ and nominal input $\bar{u}(t)$ can be regarded as additional time-varying parameters of Equation (3), we can rewrite Equation (3) as

$$
\dot{e}=F\left(\bar{x}, \bar{u}, e, u_{l c}(e)\right)=F(t, e)
$$

where $F(t, e)=f(\bar{x}+e)+g(\bar{x}+e)\left(\bar{u}+u_{l c}\right)-f(\bar{x})-g(\bar{x}) \bar{u}$.

Assumption 1 Let $x=0$ be an exponentially stable equilibrium point of the nominal system (4), where $F:[0, \infty) \times D \rightarrow \mathbb{R}^{n} \quad$ is continuously differentiable, $D=\left\{e \in \mathbb{R}^{n} \mid\|e\|<r_{0}\right\}$ and the Jaccobian matrix $[\partial F / \partial e]$ is bounded and Lipshitz on $D$, uniformly in $t$. There exists a nominal control law $\bar{u}$ and a time-varying feedback control law $u_{l c}$ such that $\dot{e}=F(t, e)$ is locally exponentially stable.

With the assumption that the tracking errors e are small by performance requirement, the tracking error dynamics can be linearized along the nominal trajectory as

$$
\dot{e}=A(t) e+B(t) u_{l c}
$$

where

$$
\begin{aligned}
& A(t)=A(\bar{x}, \bar{u})=\left.(\partial f / \partial x+\partial g / \partial x \cdot u)\right|_{\bar{x}, \bar{u}} \\
& B(t)=B(\bar{x}, \bar{u})=\left.g\right|_{\bar{x}, \bar{u}}
\end{aligned}
$$

Assumption 2 The system (5), $(A(t), B(t))$ is uniformly completely controllable.

Suppose the linearized error dynamics (5) satisfy Assumption 1 and Assumption 2. Then, there exists a LTV state feedback

$$
u_{l c}=K(t) e(t)
$$

that can exponentially stabilize the system (5) at the origin by assigning to the close-loop system the desired PD spectrum[11], where

$$
A_{z}(t)=A(t)+B(t) K(t)
$$

According to Theorem 3.11 in Ref [15], nonlinear error dynamic along the nominal trajectory is also exponentially stable at the origin. Thus, the system can be exponentially stabilized along the nominal trajectory.

The detailed design procedure and theory for PDspectrum assignment is presented in Ref [11], along with guidelines on the selection of the closed-loop PD-spectrum. According to Theorem 3.1-5.2 in Ref [11].

$$
A_{z}(t)=\left[\begin{array}{cccc}
A_{z}^{1}(t) & \mathbf{0} & \cdots & \mathbf{0} \\
\mathbf{0} & A_{z}^{2}(t) & \cdots & \mathbf{0} \\
\vdots & \vdots & \ddots & \vdots \\
\mathbf{0} & \mathbf{0} & \cdots & A_{z}^{m}(t)
\end{array}\right]
$$

where

$$
A_{z}^{i}(t)=\left[\begin{array}{ccccc}
0 & 1 & \cdots & 0 & 0 \\
0 & 0 & \ddots & \vdots & 0 \\
\vdots & \vdots & \cdots & 1 & \vdots \\
0 & 0 & \cdots & 0 & 1 \\
-\lambda_{i, 1} & -\lambda_{i, 1} & \cdots & -\lambda_{i, \mathrm{j}-1} & \lambda_{i, \mathrm{j}}
\end{array}\right]
$$

If the subsystem $A_{z}^{i}(t)$ is a second-order system, the PD spectrum $\rho_{i, k}(t)(k=1,2)$ of the $\mathrm{i}$-th second-order system is designed as

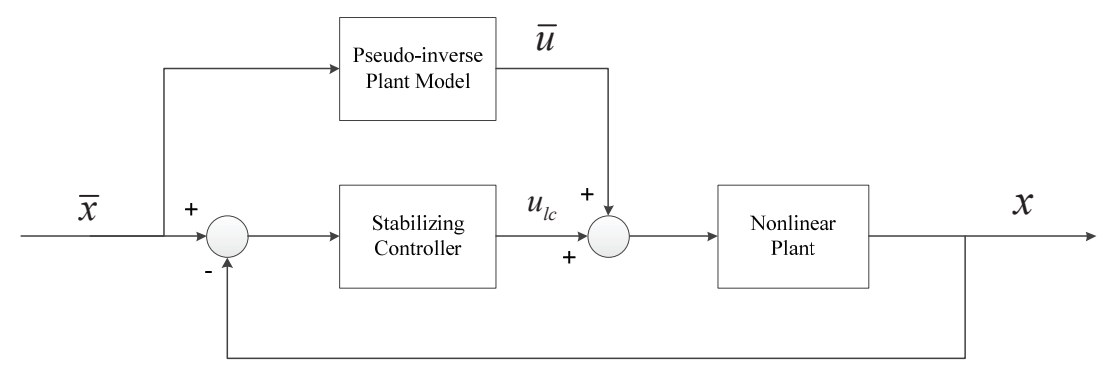

Fig. 1. Nonlinear tracking system configuration 


$$
\rho_{i, k}(t)=\left\{\begin{array}{cc}
-\left(\zeta_{i} \pm j \sqrt{1-\zeta_{i}^{2}}\right) \omega_{n i}(t) & 0<\left|\zeta_{i}\right|<1 \\
-\omega_{n i}(t),-\omega_{n i}(t)+\frac{\omega_{n i}(t)}{\int \omega_{n i}(t) d t} & \left|\zeta_{i}\right|=1 \\
-\left(\zeta_{i} \pm \sqrt{\zeta_{i}^{2}-1}\right) \omega_{n i}(t) & \left|\zeta_{i}\right|>1
\end{array}\right.
$$

where $\zeta_{i}$ is the constant damping and $\omega_{n i}(t)$ is the timevarying bandwidth. Then the time-varying coefficients of the second-order system are

$$
\begin{aligned}
& \lambda_{i, 1}(t)=\omega_{n i}^{2}(t) \\
& \lambda_{i, 2}(t)=2 \zeta_{i} \omega_{n i}(t)-\dot{\omega}_{n i}(t) / \omega_{n i}(t)
\end{aligned}
$$

\section{Governing Equations of Motion}

The realization of the MaRV-moving mass two-body system is shown in Fig. 2, and it consists of a cone-shaped body. The moving mass is allowed to translate with respect to the MaRV, but is not allowed to rotate with respect to the MaRV.

The system translational dynamics are given by Equation (10).

$$
(M+m) \dot{V}_{g}+m C_{b}^{g}\left[\omega_{b} \times\left(\omega_{b} \times P_{b}\right)+\dot{\omega}_{b} \times P_{b}+2 \omega_{b} \times \dot{P}_{b}+\ddot{P}_{b}\right]=F_{g}+G_{g}
$$

The aerodynamic force model is given by Equation (11).

$$
F=\left[\begin{array}{c}
F_{x} \\
F_{y} \\
F_{z}
\end{array}\right]=\frac{1}{2} \rho V^{2} S\left[\begin{array}{c}
c_{x 0}+c_{x}^{\alpha^{2}} \alpha^{2}+c_{x}^{\beta^{2}} \beta^{2} \\
c_{y 0}+c_{y}^{\alpha} \alpha \\
c_{z 0}+c_{z}^{\beta} \beta
\end{array}\right]
$$

The kinematic equations of attitude when the system is rolling against the centroid of the shell are given by Equation (12).

$$
\omega_{b}=\left[\begin{array}{c}
\omega_{1} \\
\omega_{2} \\
\omega_{3}
\end{array}\right]=\left[\begin{array}{ccc}
0 & \sin \vartheta & 1 \\
\sin \gamma & \cos \vartheta \cos \gamma & 0 \\
\cos \gamma & -\cos \vartheta \sin \gamma & 0
\end{array}\right]\left[\begin{array}{c}
\dot{\vartheta} \\
\dot{\psi} \\
\dot{\gamma}
\end{array}\right]
$$

The system rotational dynamics are given by Equation (13).

$$
\begin{aligned}
\left(J+\mu J_{m}\right) \dot{\omega}_{b}+\omega_{b} \times\left(J+\mu J_{m}\right) \omega_{b} & =M_{b}^{a}-\mu M P_{b} \times \ddot{P}_{b} \\
& -2 \mu M P_{b} \times\left(\omega_{b} \times \dot{P}_{b}\right)-\mu P_{b} \times F_{b}
\end{aligned}
$$

where

$$
J_{m}=m\left[\begin{array}{ccc}
q^{2}+r^{2} & -l q & -l r \\
-l q & l^{2}+r^{2} & -q r \\
-l r & -q r & l^{2}+q^{2}
\end{array}\right]
$$

and the reduced-mass parameter is given by

$$
\mu=m M /(M+m)
$$

The aerodynamic moment model is given by Equation (14).

$$
M^{a}=\left[\begin{array}{c}
M_{x}^{a} \\
M_{y}^{a} \\
M_{z}^{a}
\end{array}\right]=\frac{1}{2} \rho V^{2} S L\left[\begin{array}{c}
m_{x 0}+m_{x}^{\beta} \beta+m_{x}^{\bar{\omega}_{x}} \bar{\omega}_{x}+m_{x}^{\bar{\omega}_{y}} \bar{\omega}_{y} \\
m_{y}^{\beta} \beta+m_{y}^{\bar{\omega}_{y}} \bar{\omega}_{y}+m_{y}^{\bar{\beta}} \overline{\dot{\beta}} \\
m_{z 0}+m_{z}^{\alpha} \alpha+m_{z}^{\bar{\omega}_{z}} \bar{\omega}_{z}+m_{z}^{\bar{\alpha}} \overline{\dot{\alpha}}
\end{array}\right]
$$

where $\bar{\omega}_{x}=\frac{\omega_{x} L}{V}, \bar{\omega}_{y}=\frac{\omega_{y} L}{V}, \bar{\omega}_{z}=\frac{\omega_{z} L}{V}, \overline{\dot{\alpha}}=\frac{\dot{\alpha} L}{V}, \overline{\dot{\beta}}=\frac{\dot{\beta} L}{V}$.

Equation (10) and Equation (13) describe the mathematical model of the MaRV-moving mass two-body system. See Ref [4] and [5] for the detailed derivation for the governing equations of motion.

The roll channel dynamic equation is derived according to $\operatorname{Ref}[4]$.

Let $l=q=0$ and $J_{s}=J+\mu J_{m}$ for brevity, then

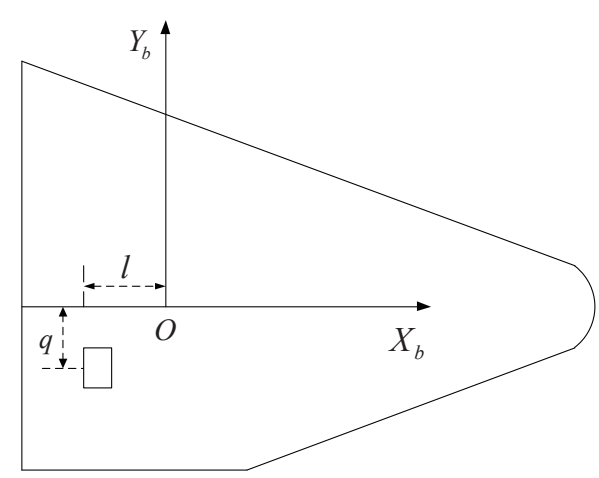

a. Side View

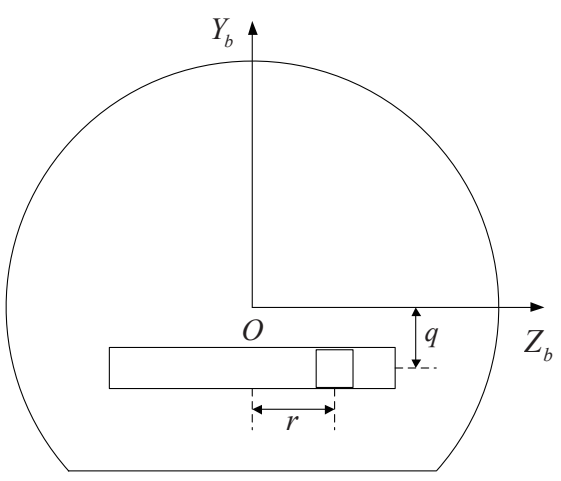

b. Rear View

Fig. 2. Coordinate-frame definitions 
$\dot{\omega}_{1}=\left[\begin{array}{lll}1 & 0 & 0\end{array}\right] J_{s}^{-1} M_{0}$

Where $M_{0}=M_{a b}-\mu M P_{b} \times \ddot{P}_{b}-2 \mu M P_{b} \times\left(\omega_{b} \times \dot{P}_{b}\right)-\mu P_{b} \times F_{b}-\omega_{b} \times J_{s} \omega_{b}$. From Equation (12) we can get

$\dot{\gamma}=\omega_{1}-\left(\omega_{2} \cos \gamma-\omega_{3} \sin \gamma\right) \tan \vartheta$

Derivate both sides of Equation (16) and substitute it into Equation (15). Then Equation (15) can be rewritten as

$\ddot{\gamma}=\left[\begin{array}{lll}1 & 0 & 0\end{array}\right] J_{s}^{-1} M_{0}+\mathrm{d}\left[-\left(\omega_{2} \cos \gamma-\omega_{3} \sin \gamma\right) \tan \vartheta\right] / \mathrm{dt}$

With further consolidation, Equation (17) is rewritten as

$$
\ddot{\gamma}=f_{f}(\gamma, \dot{\gamma})+g_{f} r+d_{s}
$$

where

$$
\begin{aligned}
& f_{f}(\gamma, \dot{\gamma})=\left(\omega_{2} \cos \gamma-\omega_{3} \sin \gamma\right) \dot{\vartheta} \csc ^{2} \vartheta \\
&-\left(\dot{\omega}_{2} \cos \gamma-\omega_{2} \dot{\gamma} \sin \gamma-\dot{\omega}_{3} \sin \gamma-\omega_{3} \dot{\gamma} \cos \gamma\right) \tan \vartheta \\
& g_{f}=\mu \frac{F_{y b}}{J_{1}} \\
& d_{s}=J_{1}^{-1}\left[\begin{array}{lll}
1 & 0 & 0
\end{array}\right]\left[M_{a b}-\mu M P_{b} \times \ddot{P}_{b}-2 \mu M P_{b} \times\left(\omega_{b} \times \dot{P}_{b}\right)-\omega_{b} \times J_{s} \omega_{b}\right]
\end{aligned}
$$

Analysis shows that the system rotational dynamic equation is non-linear, coupled and time-varying. There are also numbers of disturbing moments during the re-entry. However, the widely used classical PD control theory cannot meet the needs of MMRCS. This paper presents the attitude controller for the roll channel using TLC.

\section{MaRV Controller Design}

The controller is based on the roll channel dynamic equation (18) and the desired equation ignoring the disturbance term is rewritten as

$$
\ddot{\gamma}=f_{f}(\gamma, \dot{\gamma})+g_{f} r
$$

According to the design philosophy of TLC, it is essential to get the nominal control instruction of the system. The nominal control instruction of the system is the control instruction of the vehicle's roll angle and roll angular velocity, namely $\dot{\bar{\gamma}}=\dot{\gamma}_{C}$. The nominal position of the moving-mass is

$$
\bar{r}=g_{f}{ }^{-1}\left[\ddot{\bar{\gamma}}-\bar{f}_{f}(\bar{\gamma}, \dot{\bar{\gamma}})\right]
$$

At the same time, to ensure causality, $\ddot{\bar{\gamma}}$ and $\dot{\bar{\gamma}}$ are obtained by the following pseudo differentiator

$$
G_{\text {diff }}=\frac{\omega_{\text {diff }} s}{s+\omega_{\text {diff }}}
$$

where $\omega_{\text {diff }}$ is the bandwidth of the low pass filter. Various factors should be comprehensively considered in choosing $\omega_{\text {diff }}$ so the low pass filter can eliminate high frequency noise and allow the given control instruction.

Define the state error of system as

$$
e=x-\bar{x}
$$

According to the design philosophy of TLC, the linearized

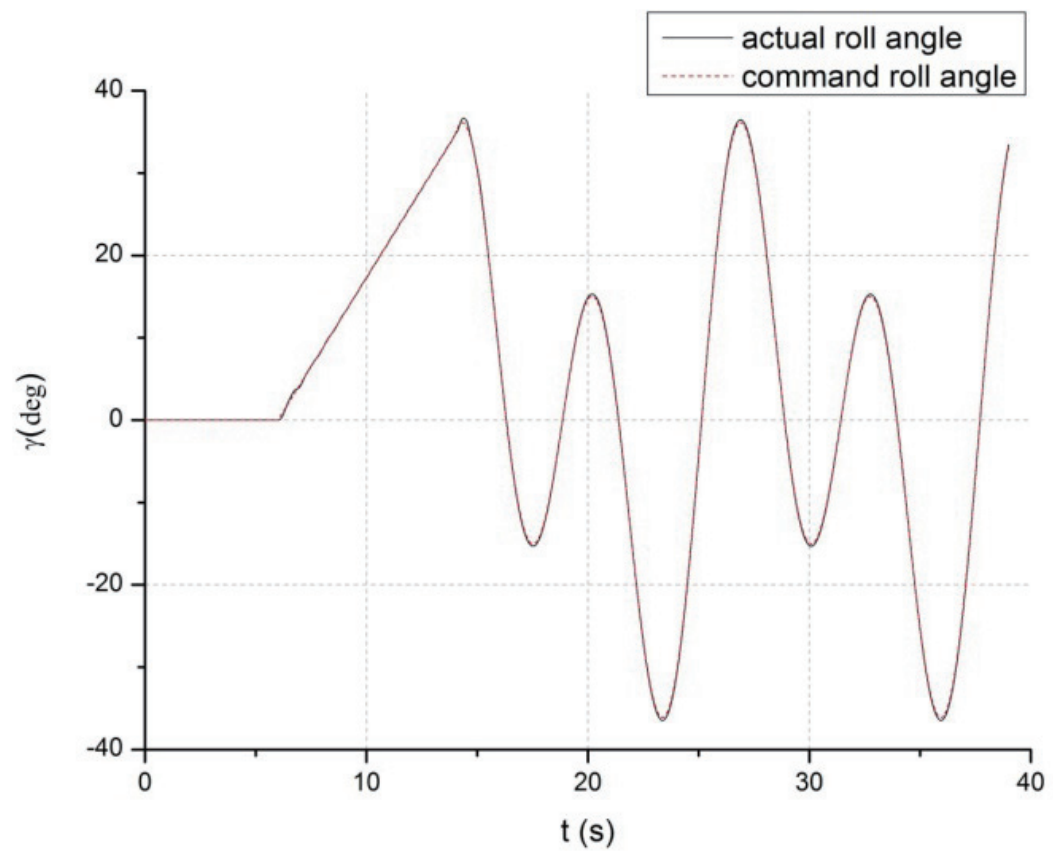

Fig. 3. Response of roll angle 
matrix of tracking error dynamics $e$ along $(\bar{x}, \bar{u})$ is

$$
A(t)=\left[\begin{array}{cc}
0 & 1 \\
a_{1}(t) & a_{2}(t)
\end{array}\right], B(t)=\left[\begin{array}{c}
0 \\
g_{f}
\end{array}\right]
$$

where

$$
\begin{aligned}
a_{1}(t) & =\left(-\omega_{2} \sin \gamma-\omega_{3} \cos \gamma\right) \dot{\vartheta} \csc ^{2} \vartheta \\
& -\left(-\dot{\omega}_{2} \sin \gamma-\omega_{2} \dot{\gamma} \cos \gamma-\dot{\omega}_{3} \cos \gamma+\omega_{3} \dot{\gamma} \sin \gamma\right) \tan \vartheta \\
a_{2}(t) & =\left(\omega_{2} \sin \gamma+\omega_{3} \cos \gamma\right) \tan \vartheta
\end{aligned}
$$

If the desired closed-loop dynamic behavior is

$$
A_{C}(t)=\left[\begin{array}{cc}
0 & 1 \\
-\lambda_{1} & -\lambda_{2}
\end{array}\right]
$$

Then according to

$$
A_{C}(t)=A(t)+B(t) K(t)
$$

The expression of $K(t)$ is

$$
K(t)=\left[\begin{array}{ll}
k_{11}(t) & k_{12}(t) \\
k_{21}(t) & k_{22}(t)
\end{array}\right]
$$

where

$$
\begin{aligned}
& k_{11}(t)=0 \\
& k_{12}(t)=0 \\
& k_{21}(t)=\left(-\lambda_{1}-a_{1}(t)\right) / g_{f} \\
& k_{22}(t)=\left(-\lambda_{2}-a_{2}(t)\right) / g_{f}
\end{aligned}
$$

The control input of the system is

$$
r_{C}=\bar{r}+K(t) e
$$

According to the pre-established PD-spectrum theory, the time-varying parameters are

$$
\begin{aligned}
& \lambda_{1}=\omega^{2}(t) \\
& \lambda_{2}=2 \zeta \omega(t)-\frac{\dot{\omega}(t)}{\omega(t)}
\end{aligned}
$$

\section{Simulation}

A numerical simulation of the full, nonlinear 6-DOF equations of motion is used to examine the time response of the TLC for the given roll command.

The initial conditions for the simulation are: initial speed $V_{0}=7000 \mathrm{~m} / \mathrm{s}$, initial height $h_{0}=50 \mathrm{~km}$, initial flight path angle $r_{0}=10^{\circ}$. The damping and time-varying bandwidth of the linear time-varying regulator are $\zeta=0.8$ and $\omega(t)=50$ for all the parameters used in the controller. The bandwidth of the low pass filter $\omega_{\text {diff }}$ is 10 . The lateral position limit of the moving mass is $\pm 0.5 \mathrm{~m}$.

The time histories of the roll angle and tracking error are shown in Fig. 3 and Fig. 4. As can be seen from the plot, the roll response is very quick with little overshoot. The maximum peak overshoot is about 0.7 degree, or $1.75 \%$ of the 40-degree commanded roll angle. Also, the tracking error is exponentially stabilized as time goes on.

The envelope values of wind speed with a $99 \%$ probability are shown in Table 1 according to Ref [16]. Simulations are

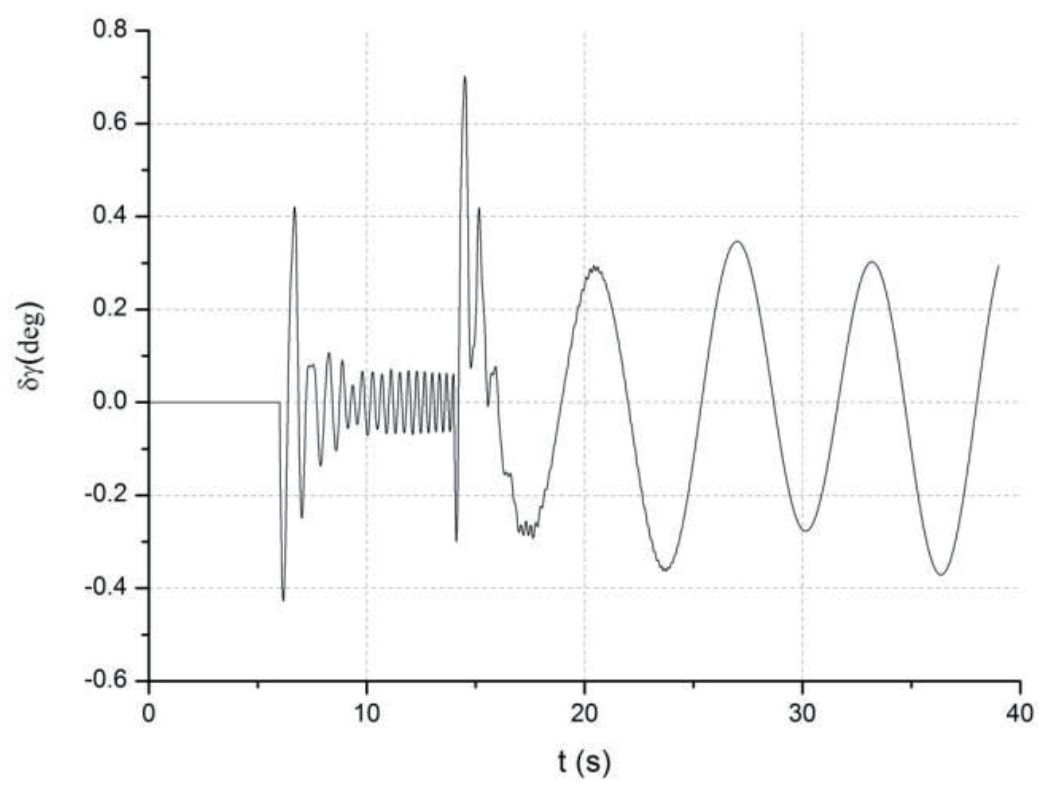

Fig. 4. Tracking error of roll angle 
performed at the same given roll command. Fig. 5 and Fig. 6 show the responses and tracking errors of roll angle with wind disturbances.

The controller is stable when there are wind disturbances.
The maximum peak overshoot of all curves is about 0.7 degree, or $1.75 \%$ of the 40 -degree commanded roll angle. Also, the tracking errors all follow the same trend when exponentially stabilized.

Table 1. Envelope values of wind speed with a $99 \%$ probability

\begin{tabular}{|c|c|c|c|c|c|c|c|c|c|c|c|c|c|c|c|c|}
\hline \multirow{2}{*}{$99 \%$} & $\mathrm{H} / \mathrm{km}$ & 1 & 3 & 5 & 7 & 9 & 11 & 12 & 13 & 14 & 15 & 20 & 30 & 40 & 50 & 80 \\
\cline { 2 - 23 } & $\mathrm{W} /(\mathrm{m} / \mathrm{s})$ & 28 & 38 & 56 & 68 & 88 & 88 & 92 & 88 & 88 & 70 & 41 & 60 & 90 & 120 & 120 \\
\hline
\end{tabular}

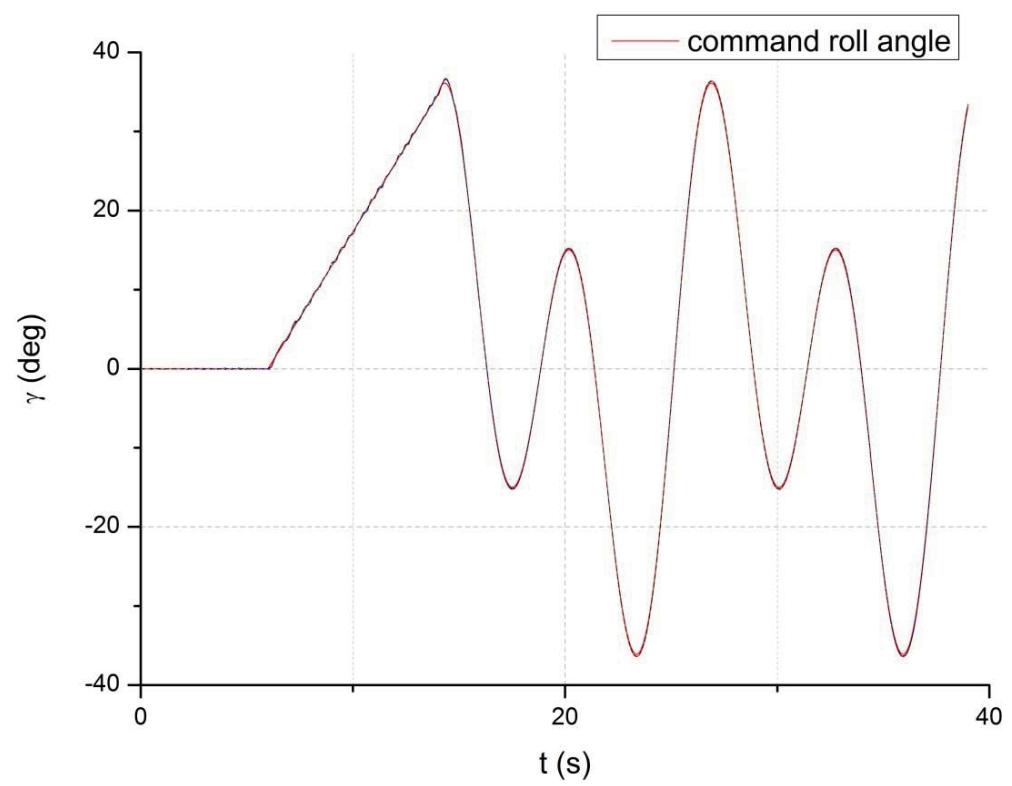

Fig. 5. Responses of roll angle with wind disturbances

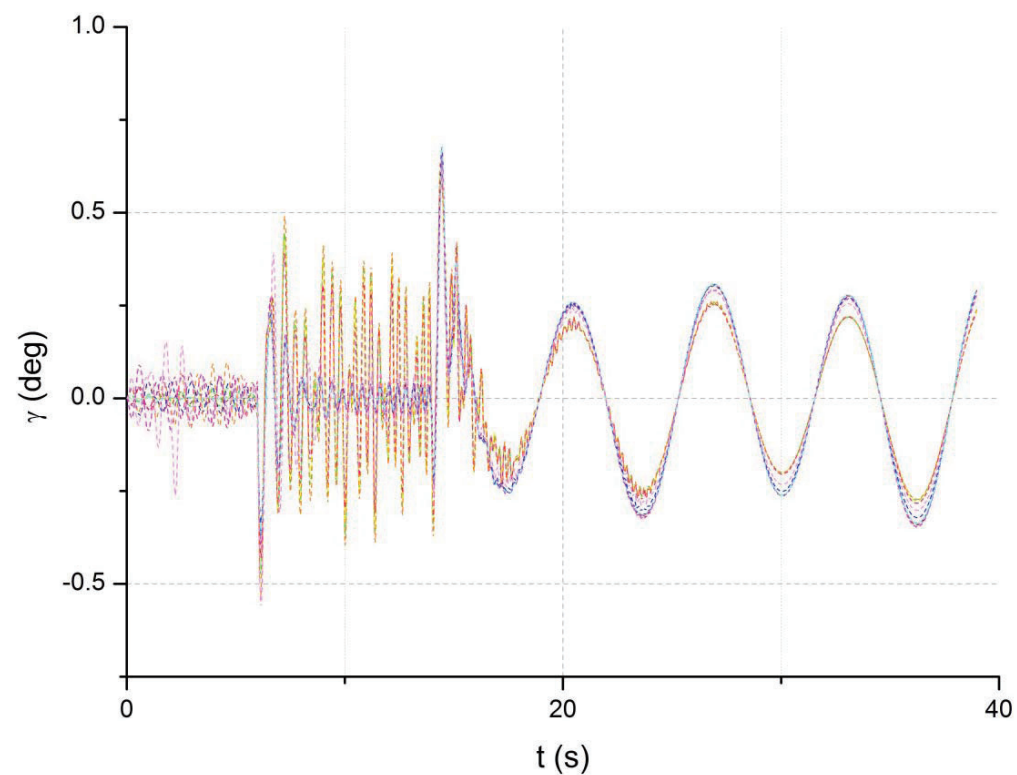

Fig. 6. Tracking errors of roll angle with wind disturbances 
Considering $\pm 30 \%$ variations in aerodynamic coefficients and $\pm 10 \%$ variations in atmospheric density, the simulations are performed at the same given roll command. Fig. 7 and Fig. 8 show the responses and tracking errors of roll angle in various aerodynamic coefficients.

Obviously, the controller is still stable when there are variations in aerodynamic coefficients. The maximum peak overshoot of all curves is about 0.9 degree, or $2.25 \%$ of the 40-degree commanded roll angle. Also, the tracking errors all follow the same trend when exponentially stabilized.

\section{Conclusion}

This paper presented a nonlinear, time-varying controller design for an MaRV using the trajectory linearization method. The nonlinearity, coupling and time-varying characteristics of the MaRV pose great challenges to the

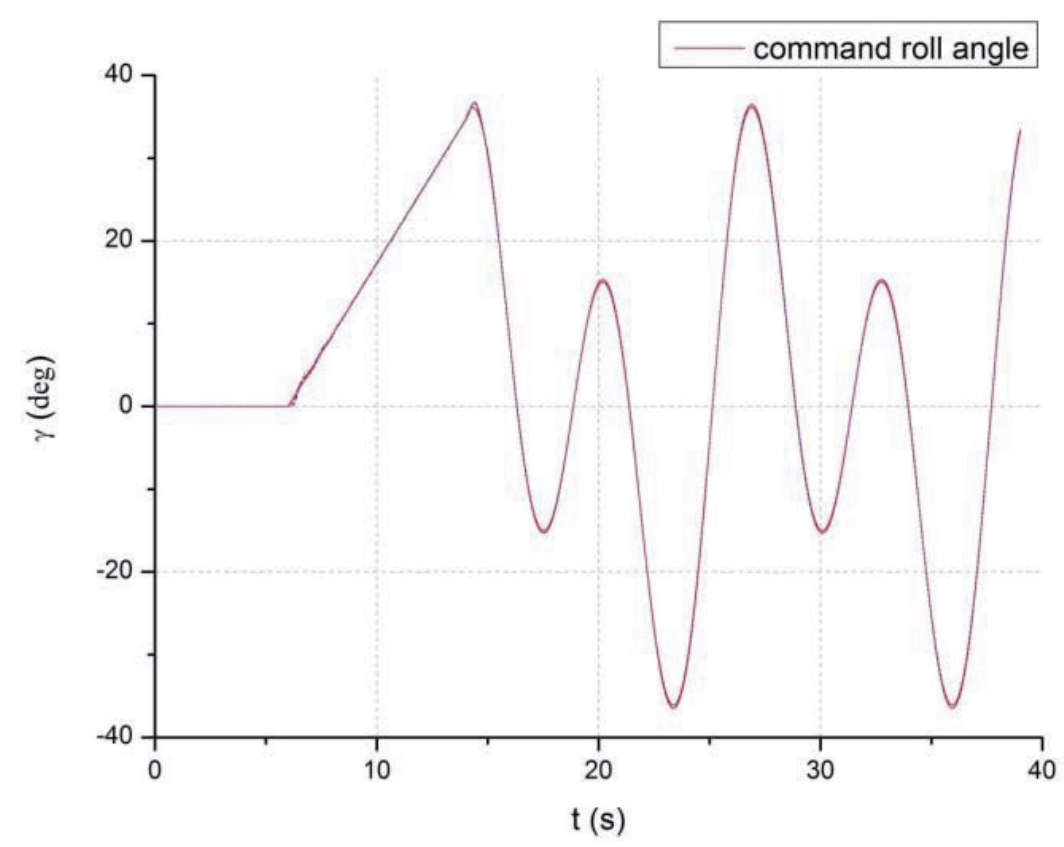

Fig. 7. Responses of roll angle in $\pm 30 \%$ variations

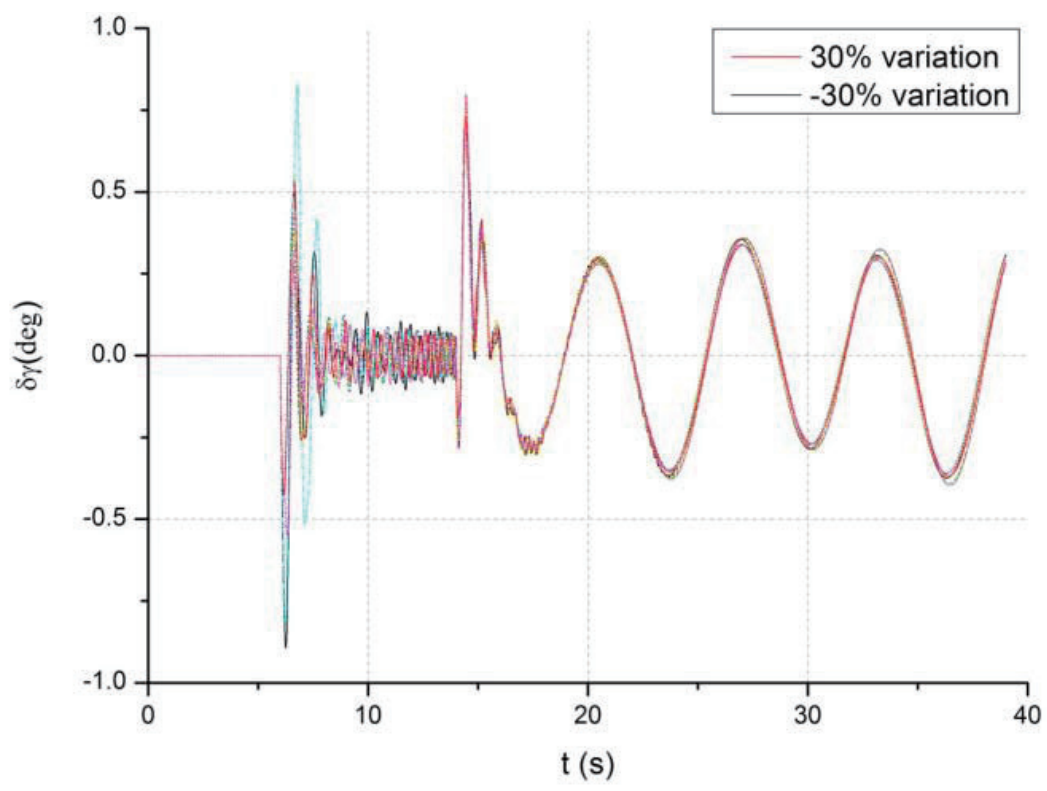

Fig. 8. Tracking errors of roll angle in $\pm 30 \%$ variations 
controller and TLC provides a satisfactory solution for the MMRCS. The controller structure exhibits considerable inherent robustness and decoupling capability without high actuator activity, providing a useful framework to deal with MaRV problems. Simulation shows that the controller is capable of dealing with different instructions. Although the controller is designed only for nominal aerodynamic coefficients, excellent performance is verified for wind disturbances and $\pm 30 \%$ variations of the aerodynamic coefficients. It is this "plug-and-play" feature that is highly preferential for developing, testing and routine operating of the re-entry vehicles.

Future research plans include improving controller performance by: (i) using a nonlinear observer to take advantage of the ignored disturbance term $d_{s}$ for a better output-feedback and (ii) $\omega_{i}(t)$ is a time-varying coefficient and time variation bandwidth (TVB) method should be taken into account. In particular, (i) should prove effective in overall tracking performance.

\section{References}

[1] Petsopoulos, Thomas, Frank J. Regan, and Jewel Barlow. "Moving-mass roll control system for fixed-trim reentry vehicle", Journal of Spacecraft and Rockets, Vol. 33, No. 1, pp. 54-60.

DOI : $10.2514 / 3.55707$

[2] Menon, P. K., G. D. Sweriduk, E. J. Ohlmeyer, and D. S. Malyevac. "Integrated guidance and control of moving-mass actuated kinetic warheads", Journal of Guidance, control, and Dynamics, Vol. 27, No. 1, pp. 118-126.

DOI:10.2514/1.9336

[3] Page, J. A., and R. O. Rogers. "Guidance and control of maneuvering reentry vehicles", Decision and Control including the $16^{\text {th }}$ Symposium on Adaptive Processes and A Special Symposium on Fuzzy Set Theory and Applications, 1977 IEEE Conference on, Vol. 16, pp. 659-664.

[4] LI Zixing and LI Gaofeng. "Moving centroid reentry vehicle modeling and active disturbance rejection roll control", Acta Aeronautica et Astronautica Sinica, Vol. 33, No. 11, pp. 2121-2129. (in Chinese)

[5] Vaddi, Sesha Sai., "Moving mass actuated missile control using convex optimization techniques", AIAA Guidance, Navigation, and Control Conference and Exhibit, Keyston, Colorado, 2006.

\section{DOI : 10.2514/6.2006-6575}

[6] Menon, P. K., S. S. Vaddi, and Ernest J. Ohlmeyer., "Finite-horizon robust integrated guidance-control of a moving-mass actuated kinetic warhead", AIAA Guidance, Navigation, and Control Conference and Exhibit, Keyston, Colorado, 2006.

DOI : $10.2514 / 6.2006-6787$

[7] Mickle, M. Chris, and J. Jim Zhu. "A nonlinear roll-yaw missile autopilot based on plant inversion and PD-spectral assignment", Decision and Control, 1998. Proceedings of the $37^{\text {th }}$ IEEE Conference on, Vol. 4, pp. 4679-4684.

[8] Mickle, M. Chris, and J. Jim Zhu. "Nonlinear missile planar autopilot design based on pd-spectrum assignment", Decision and Control, 1997., Proceedings of the $36^{\text {th }}$ IEEE Conference on, Vol. 4, pp. 3914-3919.

[9] Huang, Rui, M. Christopher Mickle, and J. Jim Zhu. "Nonlinear time-varying observer design using trajectory linearization", American Control Conference, 2003. Proceedings of the 2003, Vol. 6, pp. 4772-4778.

[10] Zhu, J. Jim, and M. Christopher Mickle. "Missile autopilot design using a new linear time-varying control technique", Journal of guidance, control, and dynamics, Vol. 20, No.1, pp. 150-157.

DOI:10.2514/2.4009

[11] Zhu, J. Jim. "PD-spectral theory for multivariable linear time-varying systems", Decision and Control, 1997., Proceedings of the $36^{\text {th }}$ IEEE Conference on, Vol. 4, pp. 39083913.

[12] Liu, Y., Wu, X., Jim Zhu, J., \& Lew, J. “Omni-directional mobile robot controller design by trajectory linearization." American Control Conference, 2003. Proceedings of the 2003, Vol. 6, pp. 3423-3428.

[13] Zhu, J. Jim, D. Banker, and Chahes E. Hall. "X-33 ascent flight control design by trajectory linearization-a singular perturbation approach." AIAA Guidance, Navigation, and Control Conference and Exhibit, Denver, Colorado, 2000.

DOI : $10.2514 / 6.2000-4159$

[14] Liang, Zhu, et al. "New trajectory linearization control for nonlinear systems undergoing harmonic disturbance", Systems Engineering and Electronics, Journal of, Vol. 20, No. 3, pp. 571-576.

[15] Kahlil, Hassan K., Nonlinear Systems 2nd ed., PrmticeHall, Upper Saddle River, NI, 1996.

[16] Johnson, Dale Leroy, “Terrestrial environment (climatic) criteria guidelines for use in aerospace vehicle development, 1993 revision." NASA-TM-4511, 1993. 